\title{
Erratum to: Prevalence of diabetes and impaired fasting glucose in the Chinese adult population: International Collaborative Study of Cardiovascular Disease in Asia (InterASIA)
}

D. Gu $\cdot$ K. Reynolds $\cdot$ X. Duan $\cdot$ X. Xin $\cdot$ J. Chen $\cdot$

X. Wu $\cdot$ J. Mo $\cdot$ P. K. Whelton $\cdot$ J. He •

for the InterASIA Collaborative Group

Published online: 16 August 2012

(C) Springer-Verlag 2012

Erratum to: Diabetologia

DOI 10.1007/s00125-003-1167-8

Age-specific numbers were inadvertently switched between the 'Undiagnosed diabetes' and 'Impaired fasting glucose' columns in Table 3. The corrected section of the table is reproduced here.

The online version of the original article can be found at http://dx.doi.org/ $10.1007 / \mathrm{s} 00125-003-1167-8$.

D. Gu $\cdot$ X. Duan $\cdot$ X. Wu

Cardiovascular Institute and Fuwai Hospital of Chinese Academy

of Medical Sciences, Peking Union Medical College,

Beijing, China

K. Reynolds $\cdot$ X. Xin $\cdot$ P. K. Whelton $\cdot$ J. He $(\bowtie)$

Department of Epidemiology,

Tulane University School of Public Health and Tropical Medicine,

1430 Tulane Avenue SL18,

New Orleans, LA 70112, USA

e-mail: jhe@tulane.edu

J. Chen · P. K. Whelton · J. He

Tulane University School of Medicine,

New Orleans, LA, USA

J. Mo

Pfizer Inc,

New York, NY, USA 
Table 3 Prevalence of undiagnosed diabetes and impaired fasting glucose ${ }^{\mathrm{a}}$ in the adult population aged 35-74 Years in China, 2000-2001

\begin{tabular}{|c|c|c|c|c|c|c|}
\hline \multirow[t]{2}{*}{ Age, yrs } & \multicolumn{2}{|l|}{ Men } & \multicolumn{2}{|l|}{ Women } & \multicolumn{2}{|l|}{ Total } \\
\hline & $\begin{array}{l}\text { Undiagnosed } \\
\text { Diabetes, \% (SE) }\end{array}$ & $\begin{array}{l}\text { Impaired Fasting } \\
\text { Glucose, \% (SE) }\end{array}$ & $\begin{array}{l}\text { Undiagnosed } \\
\text { Diabetes, \% (SE) }\end{array}$ & $\begin{array}{l}\text { Impaired Fasting } \\
\text { Glucose, \% (SE) }\end{array}$ & $\begin{array}{l}\text { Undiagnosed } \\
\text { Diabetes, \% (SE) }\end{array}$ & $\begin{array}{l}\text { Impaired Fasting } \\
\text { Glucose, \% (SE) }\end{array}$ \\
\hline \multicolumn{7}{|l|}{ North } \\
\hline $35-44$ & $3.2(0.5)$ & $8.7(1.0)$ & $3.7(0.6)$ & $5.1(0.8)$ & $3.4(0.4)$ & $7.0(0.7)$ \\
\hline $45-54$ & $3.3(0.7)$ & $7.6(1.1)$ & $5.5(0.9)$ & $6.6(1.0)$ & $4.4(0.6)$ & $7.1(0.7)$ \\
\hline $55-64$ & $5.8(1.2)$ & $8.8(1.4)$ & $5.6(1.1)$ & $11.2(1.6)$ & $5.7(0.8)$ & $10.0(1.1)$ \\
\hline $65-74$ & $3.9(1.3)$ & $12.2(2.4)$ & $10.0(1.8)$ & $9.1(2.0)$ & $6.9(1.1)$ & $10.7(1.6)$ \\
\hline $35-74$ & $3.8(0.4)$ & $8.8(0.6)$ & $5.3(0.5)$ & $7.1(0.6)$ & $4.6(0.3)$ & $8.0(0.4)$ \\
\hline Age-Standardized ${ }^{\mathrm{b}}$ & $4.3(0.4)$ & $9.1(0.6)$ & $5.7(0.4)$ & $6.8(0.5)$ & $5.0(0.3)$ & $7.9(0.4)$ \\
\hline
\end{tabular}

